ISSN: 1130-3743 - e-ISSN: 2386-5660

DOI: http://dx.doi.org/10.14201/teoredu20162825373

\title{
LA EDUCACIÓN MORAL EN CONTEXTOS INFORMALES
}

\author{
Moral education in informal contexts
}

\section{L'éducation morale dans les contextes informels}

\author{
Zaida ESPINOSA ZÁRATE \\ Universidad Internacional de La Rioja. \\ Gran Via Rey Juan Carlos I, 41. 26002 Logroño (La Rioja). \\ zaida.espinosa@unir.net
}

Fecha de recepción: enero de 2016

Fecha de aceptación: marzo de 2016

RESUMEN

En tanto que la transmisión tiene lugar de manera preeducativa, examinamos las virtualidades educativas de los contextos informales: su inmediatez respecto al mundo de la vida y su carácter multiplicador de los resultados educativos. Se reflexiona acerca del valor de la experiencia y, en consecuencia, del contexto informal como fuente de aprendizajes, observando que el ser humano no es simplemente un ser de experiencias, sino capaz de aprovecharlas o desaprovecharlas, aprender o no de ellas. Es decir, pedagógicamente hablando, la pura acumulación de experiencias tiene un potencial educativo limitado, por lo que hay que desarrollar estrategias que permitan convertirla en recurso de aprendizaje. En este sentido se advierte la reflexividad como fundamental. La base que constituye la condición de posibilidad de la traducibilidad entre contextos formales e informales -cuya convergencia refuerza enormemente los aprendizajes- es el hecho de que ambos comparten un mismo tejido moral, intrínseco a las acciones humanas.

Palabras clave: contexto de aprendizaje informal; experiencia; educación moral; reflexividad. 


\section{SUMMARY}

Since transmission takes place pre-educationally, we can be certain about the educational potential that informal contexts offer on account of the immediacy of the experience of the world they provide, and due to the multiplier effect of educational results that they have. We reflect on the value of experience and, consequently, of informal contexts as contributors to learning, realizing that humans are not just characterized by the experiences they live, but by their being capable of either making the most of them or not. Educationally speaking, the sheer accumulation of experiences has just limited educational potential. Therefore, we need to develop strategies that turn them into valuable learning contributors. A reflexive character turns out to be essential. The basis that serves as a condition for the affinity of both contexts -whose confluence reinforces greatly learning processes- is the fact that both share the same moral fabric, intrinsic to human actions.

Key words: informal learning contexts; experience; moral education; reflexive character.

\section{SOMMAIRE}

Tant que la transmition a lieu de façon pre-éducative, nous examinons les vertus éducatives qui presentent les contextes informels: sa proximité au regard du monde de la vie, et son caràctere multiplicateur des résultats éducatifs. On reflechit autour de la valeur de l'expérience et, en conséquence, du contexte informel comme source d'aprentissage, en observant que l'être humain n'est pas simplement un être d'expériences, mais un être capable de mettre à profit ou pas, apprendre o ne pas le faire d'elles. C'est à dire, pédagogiquement parlant, le simple cumul d'expériences a un potentiel éducatif limité, ce pour quoi il faut developper des strategies qui permettent de le convertir en précieuses sources d'apprentissage. La base qui constitue la condition de possibilité de la traductibilité entre les contextes formels ou informels -dont la convergence renforce fortement les apprentissages-, est le fait que tous deux partagent un même tissu moral, intrinsèque aux actions humaines.

Mots clés: contextes d'apprentissage informel; expérience; education morale; reflexivité.

1. El FENÓMENO UNIVERSAL DE LA TRANSMISIÓN Y EL CARÁCTER PERMANENTEMENTE COMPRENSIVO E INTERPRETADOR DEL SER HUMANO

El ser humano habita un mundo que es cultura, y esto significa constructo, artefacto alejado de la naturaleza primera, bruta u originaria. Es un mundo cultural caracterizado por la diversidad y la pluralidad que constituyen la riqueza de la existencia humana y son expresión de su libertad; algo que, siguiendo a Gehlen, puede caracterizarse como una segunda naturaleza (Gehlen, 1980). Todo lo demás, lo 
natural como lo opuesto a lo artificial (en el sentido de no transformado), es ajeno a lo humano, resulta humanamente inhabitable hasta que ha experimentado cierta transformación. Ésta es fruto de la capacidad artística del hombre y está marcada por el carácter simbólico que es propio de la existencia humana y del mundo que habita $^{1}$, y su naturaleza simbólica implica inmediatamente el hecho de la transmisión. El fenómeno de la transmisión -y la comprensión de significados que lleva asociada- es constante en la vida humana. Es algo que ocurre, que tiene lugar de manera no sólo explícita o intencionada, fruto de la voluntad expresa del sujeto que la provoca, sino también al margen e -incluso- en contra de su voluntad, y también por parte de objetos.

El ambiente mismo como realidad no intencional, sin obrar intencionalmente por fines, es de esta forma expresivo, transmisor. De alguna manera, toda la amplitud de lo real apela al sujeto humano, y no sólo la realidad humana (subjetiva, u objetivada en productos que son resultado de la transformación, del trabajo del hombre), sino también la natural. Es decir, no sólo es otra subjetividad, otra humanidad la que me apela, ni tampoco únicamente objetivaciones, construcciones que son resultado del hacer o producir del hombre, sino también la misma realidad natural-material ahí dada con la que me encuentro. Esta apelación encuentra a veces como respuesta la admiración como tensión intelectual suscitadora del sentir y pensar humano que demanda nuevas respuestas. En consecuencia, el comprender que aquella transmisión lleva asociado se da constantemente. Está conectado, en palabras de Gadamer, antes con un modo de ser que con una operación cognoscitiva concreta, un método o modo particular de comportamiento del hombre entre muchos otros posibles (Gadamer, 1988): es aquello que nos constituye ontológicamente, aquello que somos: la condición humana.

Es decir, la comprensión desempeña un papel en el terreno de la existencia vivida, natural, pre-científica y, podríamos añadir, pre-educativa (entendiendo aquí por educación la institucional o formal $)^{2}$. Se podría decir que estamos instalados en el lenguaje, por lo que lo originario no es enmudecer, sino dialogar, comprender, pensar juntos, proceso en el que acontece la verdad de una manera que escapa en cierta medida al control del individuo. Gracias al lenguaje estamos instalados en un ámbito común de intersubjetividad. Es decir, partimos del hecho de la

1. A raíz de esto, se plantea la pregunta de si el ser humano se reconoce en ese mundo que ha originado; es decir, si esos productos resultado de su capacidad artística tienen la virtualidad de ofrecer claves para la comprensión de la propia subjetividad (como piensa, por ejemplo, Ricoeur, al decir que la subjetividad no puede comparecer del todo a sí misma, pero se reconoce a través de sus obras; de manera que, para conocerla a ella, hay que salir fuera de ella), o si ocurre lo contrario y no hay posibilidad de reconocimiento de la subjetividad en aquello, y la salida a los objetos constituye una especie de autoalienación o extrañación paradójica de la conciencia respecto de sí misma (paradójica en tanto que tienen su origen en ella misma).

2. "Comprender e interpretar textos no es sólo una instancia científica, sino que pertenece con toda evidencia a la experiencia humana en el mundo", pues es la experiencia que nos sustenta y de la que nos nutrimos (GADAMER, 1988, 23). 
transmisión, y esto constituye, según Gadamer, la garantía de la tradición, esto es, de su pervivencia.

Respecto a esto se plantean muchas preguntas sobre las que habría que pensar: ¿cómo se da la transmisión? Es decir, se plantea la necesidad de hacer un examen, una indagación de los límites de este fenómeno. Es solo una vez establecido esto, cuando puede profundizarse en el ser mismo de la comprensión. ¿Qué es comprender? ¿Es fusión de horizontes diversos, apertura de sentidos, de posibilidades de exploración, es lo que nos muestra y nos permite tener un mundo? Se plantea también el problema, de tipo pedagógico, de la incorporación de lo comprendido al propio ser del que comprende. G. Steiner se deja asombrar por estos aspectos, por "la maravilla de la transmisión", por "el misterio que le es inherente», al que describe con tintes mágicos, incluso místicos, sin eludir el carácter problemático que presenta: "¿Qué es lo que confiere a un hombre o a una mujer el poder para enseñar a otro ser humano?» (Steiner, 2011, 11).

Se puede entender el mundo entero, en toda la amplitud de su rango material, como lenguaje. Así se dota a éste de un carácter trascendental ${ }^{3}$. Es el constitutivo de nuestro propio experimentar el mundo, el mediador universal de nuestro acceso a lo real, fuera del cual no podemos situarnos. Esto convierte a todo lo que hay, al menos en ese sentido físico-material, en signo, que es así siempre para un sujeto humano.

Es decir, la transmisión se produce de manera continua por el carácter peculiar de la subjetividad humana como un ser permanentemente interpretador, comprensivo, dotador de sentido de todo aquello en lo que pone la atención (y de aquello que, aunque no sea centro o foco de su intención gnoseológica, constituye un círculo más amplio no intencional que actúa como fondo -tras-fondo- del espíritu atento) (Husserl, 1962). Lo que nos interesa en este caso es el carácter eminentemente pedagógico, educativo de esa transmisión, que se da mucho antes de entrar a la escuela, en tanto que la comprensión que lleva aparejada constituye, como hemos visto, el ser mismo del hombre. Por tanto, esa transmisión con virtualidad educativa ocurre en demasiadas ocasiones -quizá en más de las esperadas... o incluso deseadas- de manera no intencionada, con consecuencias importantes para las acciones con intención, ahora sí, expresamente educativa.

El énfasis en la idea del aprendizaje continuo, a lo largo de toda la vida (lifelong learning), muestra una conexión lógica con el concepto, ya desde hace años reconocido desde el campo de la pedagogía, de educación en contextos informales. Es decir, ambas cosas no pueden desligarse. La educación formal, institucionalizada, estructurada y conducente a la obtención de algún reconocimiento en forma de certificado se ha expandido, diversificado y extendido enormemente en el mundo actual (ya no hay separación tajante entre el mundo de la formación, por una parte, y el del trabajo en la vida real, por otra -como la que se da en la

3. Otra cuestión distinta, no implicada en esta afirmación, es la de si esto -lenguaje- es todo lo que hay, en el sentido de que no hay nada más que eso. 
vida de un coche recién fabricado que sale nuevo, todavía sin estrenar, del garaje para empezar a ser utilizado). Esto es debido a la extensión de la obligatoriedad de la escuela secundaria y, más recientemente, a los medios digitales que permiten la flexibilización en tiempos y espacios del fenómeno educativo. Pero, si bien esto es cierto, hay que reparar también en que habitualmente la educación formal no se prolonga durante toda la vida; es decir, no suele ser continua en el tiempo. Es un hecho: por regla general no estamos matriculados en instituciones educativas de manera permanente. Sin embargo, si se afirma que el aprendizaje es permanente, no queda más remedio que reconocer la existencia, por otro lado de sentido común, de aprendizajes en contextos informales.

\section{El CARÁCTER OMNIPRESENTE DE LA EDUCACIÓN MORAL}

Hay algo que todos los contextos de aprendizaje comparten, hasta aquellos que no han sido diseñados con ninguna finalidad educativa, y es que en ellos está implicada la esencia de la educación: su dimensión moral. En tanto que ésta no es ajena a ningún acto humano, en todos los contextos se pone en juego el carácter, el ethos, las costumbres de los implicados, que se reflejan de manera integrada en el ejemplo de la(s) persona(s) que se ve actuar o en la actividad cotidiana y el propio quehacer del docente. El ejemplo (que suele ir acompañado o desencadena una especie de inercia imitativa) está en juego en todo contexto de aprendizaje, y aquellos se encuentran y se extienden a todos los escenarios de la vida. Ya Aristóteles entendió la imitación como la fuente o recurso educativo por excelencia, y en vista de esto propugnó la representación de acciones virtuosas (no simplemente de hombres virtuosos, como se observa si se lee detenidamente su Poética) ${ }^{4}$ como medio para la mejora moral del espectador, precisamente por el impulso imitativo que está contenido en la condición humana. También la virtualidad del ejemplo es lo que le hace a Steiner concebir incluso la posibilidad de una educación muda, puramente mostradora u ostentativa: «La enseñanza ejemplar es actuación y puede ser muda. Tal vez deba serlo... La enseñanza válida es ostensible. Muestra. Esta "ostentación", que tanto intrigaba a Wittgenstein, está inserta en la etimología: el latín dicere, "mostrar" y, sólo posteriormente, "mostrar diciendo"» (Steiner, 2011, 13).

Hay un aspecto vivencial que está implicado en el estudio y aprendizaje de toda materia o disciplina, desde los niveles más básicos hasta los superiores. Podríamos distinguir en todo proceso de enseñanza-aprendizaje, en todo proceso educativo -esté referido a la disciplina que sea-, dos vertientes: por una parte, una dimensión teórica, más o menos técnica, que resulta obvia en todos los ámbitos del saber (incluso los saberes más alejados de lo técnico pueden ser abordados de

4. «El más importante de estos elementos es la estructuración de los hechos; porque la tragedia es imitación, no de personas, sino de una acción y de una vida, y la felicidad y la infelicidad están en la acción, y el fin es una acción, no una cualidad» (ARISTóteles, 1974, 147). 
manera técnica, en tanto que su aprendizaje supone también una parte o aspecto de este tipo) y, por otra, una dimensión de apropiación o comprensión que el hombre hace no sólo de aquella materia, sino de la realidad que le rodea en general, que también supone -inobjetiva o concomitantemente, empleando la terminología de Millán-Puelles- una apropiación o comprensión de sí mismo. Esta última es una cuestión que no es puramente teórica ni técnica, sino vivencial, y en este sentido puede hablarse de todas y cada una de las disciplinas como modos de vida.

Este aspecto vivencial que está implícito en el aprendizaje y comprensión de las disciplinas desde los niveles más básicos exige un concepto de educación amplio que ya ha sido recuperado desde el mundo académico (Ibáñez-Martín, 2013) y que engloba la dimensión moral que es intrínseca a la vida humana, a la acción humana -también la profesional-. Maxwell y otros (Maxwell, TremblayLaprise, Filion, Boon, Daly, Van den Hoven, Heilbronn, Lenselink y Walters, 2016) apuntan a los interrogantes que se plantean a la hora de decidir el modelo (integrado o independiente) más efectivo para enseñar ética a los estudiantes que están preparándose para ser profesores, pues de esto dependerá la calidad futura de su docencia y su éxito al enfrentarse a los retos éticos que plantea la docencia contemporánea, y esto puede extenderse a cualquier actividad profesional. Es decir, ese aspecto vivencial al que nos referimos supone, por una parte, que toda labor educativa integral en contextos formales acarrea necesariamente la tarea de plantear y profundizar en cuestiones que tienen que ver con el tejido moral de la actividad a la que se refieren, en tanto que ésta es una actividad humana. Y, por otra parte, implica que la acción educativa se da ya en la vida misma, de manera previa a todo acceso a un sistema de educación formal.

Del lado de la educación formal, esto tiene como consecuencia que, además del progreso material o técnico en los resultados alcanzados por el aprendiz de cada disciplina gracias a la ayuda del profesor -que constituye una parte fundamental de la labor de enseñanza-, también hay otro aspecto que el propio docente debe cultivar (plantar, poner la semilla) en el alumno o alumna, en tanto que el estudio y aprendizaje de una disciplina consiste también en la apropiación de un modo de vida -y tiene, por tanto, un carácter intrínsecamente moral-. "Afortunado el discípulo cuyo Maestro ha dado sentido a la moral», dice Steiner (Steiner, 2011, 59). Esto puede verse en la cierta connaturalidad que tienen los miembros pertenecientes a la misma escuela, marcados por un mismo modo de vida -piénsese, por ejemplo, en la escuela pitagórica, entre muchos otros ejemplos-, los discípulos que siguen a un mismo maestro, con deseos de asemejarse o parecerse cada vez más a él, aunque luego aparezca la posibilidad, siempre presente y efectivamente dada a lo largo de la historia, de la traición, la afirmación de uno mismo al margen del maestro, o su superación (Steiner, 2011). Es decir, el profesor ha de ejercer como guía para el educando para que éste avance por un camino -la adquisición de ese modo de vida- que tiene que recorrer por sí solo (pues la vida sólo puede llevarse o dirigirse $-\mathrm{O}$ no hacerlo- en primera persona), y en el que se realizará como persona humana, sin que quepa deslindar el ámbito público -de la profesión- de la vida privada. 
Así pues, aunque en los procesos de enseñanza-aprendizaje de disciplinas técnicas lo que suele importar, lo que más cuenta, es el resultado o el efecto conseguido en el estudiante a través de la acción docente -resultados medibles en términos cuantitativos, de cantidad de información o de habilidad o competencia desarrollada-, no hay que olvidar la importancia del camino, del propio proceso -la adquisición de unos modos de proceder, de unos hábitos que han calado en el alumno al menos en cierta medida- que le ha conducido a esos resultados, que constituye la dimensión moral inserta en la tarea educativa y que tiene más calado, alcanza un nivel de profundidad superior que los resultados mismos que, cuando no son entrenados, se olvidan y pierden con facilidad.

Conviene detenernos un momento a profundizar en el carácter omniabarcante de la educación moral. Su operatividad constante radica en el hecho de que lo impregna todo, en al menos tres sentidos que pueden distinguirse: primero, se da en todos los contextos de aprendizaje; segundo, en o respecto a todas las materias, está referida a todos los objetos (no sólo a los estrictamente morales: las acciones inmanentes), y, por último, afecta a todos los sujetos, independientemente de su función en la escala social.

Por tanto, no se restringe o limita a los aprendizajes adquiridos en clases de ética o ética aplicada (entendiendo la ética como una reflexión teórica acerca del fenómeno moral), ni a aquellos que versan sobre asuntos u objetos estrictamente morales (acciones humanas inmanentes, frente a las transeúntes o puramente técnicas), sino que puede entenderse como dando cabida al proceso de formación de todo juicio reflexivo que nos hace capaces de convertirnos en jueces de nuestra propia conducta (Green, 1985). Y ésta ha de entenderse de manera amplia, abarcando también cuestiones técnicas o artísticas (el sentido moral me permite medir en qué medida se ajustan mis resultados técnicos a los estándares de esa práctica, si bien la racionalidad implicada en este caso es una racionalidad instrumental) ${ }^{5}$, sociales (pues la formación de la conciencia sólo se da en un contexto social y referida a él, siendo conciencia de pertenencia, frente a la consideración habitual hoy ampliamente extendida de la educación moral como centrada únicamente en el individuo $)^{6}$, históricas, etc. En este sentido considera Green que la conciencia moral tiene varias voces, y su educación implica atender a todas ellas (Green, 1985).

\section{LAS VIRTUALIDADES EDUCATIVAS DE LOS CONTEXTOS INFORMALES}

Si bien, como ha sido reconocido ampliamente, la dimensión moral está presente en toda tarea explícitamente educativa (el profesor de matemáticas no

5. Hay que notar, como hace Aristóteles en la Ética a Nicómaco, que lo técnico y lo moral no coinciden en ciertos respectos: por ejemplo, el que yerra voluntariamente en asuntos del arte es mejor o muestra mayor destreza que el que lo hace sin querer, frente a lo que ocurre en las acciones morales.

6. «La conciencia como pertenencia es la realidad fundamental que la educación moral debiera tomar como objeto» (GREEN, 1985, 8). 
sólo enseña matemáticas, sino un modo de ver y afrontar la realidad), aquí se trata de reparar en la enorme potencialidad que ofrecen los contextos informales de aprendizaje a este respecto en comparación con los entornos de aprendizaje específicamente concebidos y diseñados para esta tarea. De hecho, el aprendizaje formal no debe entenderse al margen de este otro tipo de aprendizajes en contextos informales, pues éstos resultan a nivel moral incluso más moldeadores del carácter, de las costumbres (ethos) del individuo, que la educación formal en numerosas ocasiones. Por ello, nos proponemos señalar sus principales beneficios en el aspecto que estamos considerando.

\subsection{La inmediatez del contexto informal vs. la separación del aula respecto del mundo de la práctica}

Los contextos informales tienen un efecto multiplicador del potencial educativo en lo que respecta a la formación moral por su propio carácter más próximo o imbuido en el mundo de la vida, por la ausencia de separación que el estudiante percibe en ellos respecto de la vida, es decir, por la propia sensación del estudiante de estar actuando en un contexto real.

Esta visión se asienta sobre la base de considerar que el mundo y la cultura educativos, las instituciones y centros designados específicamente para llevar a cabo la función educadora de los ciudadanos, llevan inserta en sí mismos una disociación respecto de la práctica, del mundo de la vida, que, por otra parte, cada vez se percibe como más problemática y acuciante. No faltan las denuncias respecto a esta quiebra, que da lugar, según algunos, a profesionales no preparados para las exigencias de la vida laboral. Es decir, se denuncia la educación formal por su cierta irrelevancia para la práctica. Se plantea lo que Schön denomina «el dilema del rigor o la pertinencia” (Schön, 2010, 21). En el desempeño práctico del profesional no se dan las dos a la vez: o bien se opta por el rigor en el ejercicio profesional, siguiendo el modo de proceder de la racionalidad científico-técnica, pero entonces uno se encuentra que este modelo resulta ineficaz o simplemente inaplicable en contextos prácticos reales por las características difusas o problemáticas de éstos; o bien se opta por la pertinencia de las acciones que uno lleva a cabo en la práctica, cosa que resulta incompatible con el rigor, en tanto que, cuando el profesional experto hace algo que resulta relevante para el contexto específico que afronta, no lo hace siguiendo rigurosamente las reglas de un método científico-técnico que ha aprendido teóricamente al estudiar las disciplinas básicas que constituyen la base de su práctica profesional, sino que sus acciones son fruto de un arte que ha adquirido en la práctica misma, no separadamente de ella ("conocimiento en la acción", lo denomina Schön) (Schön, 2010, 35). Esta brecha abierta ha sido denunciada por muchos. Knight y otros lo hacen -entre otros- en el campo de la formación del profesorado: «Findings revealed the very different, often conflicting views of teaching and learning that universities espouse in contrast to teaching and learning implemented in schools and/or mandated by education policies» (Knight, 
Lloyd, Arbaugh, Gamson, McDonald, Nolan y Whitney, 2015). Por ello se reconoce como más urgente que nunca la necesidad de establecer puentes entre el mundo de la formación y, en concreto, de la universidad -por ser la institución educativa que debiera capacitar de manera más directa para el desempeño profesional- y el de la práctica profesional (Ethel y McMeniman, 2000).

No les falta razón, pues, como también afirma Schön (Schön, 2010, 19), en las prácticas educativas, en los prácticums a los que asisten los alumnos habitualmente en los últimos cursos de carrera (que son los contextos en los que se encuentran más cercanos al mundo de la vida), todavía la práctica con la que se enfrenta el estudiante está diseñada para que él pueda asumirla y, por tanto, carece de los riesgos que se dan en la vida real. Es decir, ha sido modulada y ajustada a las capacidades todavía incipientes del que se está iniciando en el desempeño de esa práctica profesional.

La percepción por parte del estudiante que se encamina al centro educativo, ya sea de educación superior o secundaria, es de falta de contacto de aquello que va a hacer con respecto al mundo real. Es decir, lleva consigo la conciencia de su separación respecto al mundo de la vida, sabe que no se va a enfrentar a nada auténticamente real. En cambio, el aprendizaje informal tiene el potencial de eliminar esa fractura que el propio estudiante percibe de forma peyorativa por su cierta falsedad, su carácter construido, artificialmente moldeado y diseñado, aunque este constructo tenga la finalidad precisamente de facilitarle y acelerarle su proceso de aprendizaje.

Es el carácter de inmediatez, el establecimiento de un contacto directo con lo real, el multiplicador del potencial educativo y transmisor de conocimientos de los contextos informales. Por mucho que no lo parezca, a juzgar por el manejo constante de mediaciones tecnológicas por parte del joven actual, el alumno está cansado de las mediaciones. El estudiante está deseoso de salir de su medio ambiente más habitual, el aula, y acceder al mundo de la vida, a toda la amplitud de las experiencias que proporciona el mundo real. Por muchas transformaciones que experimente el aula, a través de su inversión o de la adopción de otro tipo de estrategias educativas novedosas, sigue siendo aula, y el alumno la continúa percibiendo y reconociendo como tal. Lo que demanda constantemente, lo que pide a gritos es el contacto directo, la adrenalina de estar actuando, jugándosela en el mundo real, el salto al mundo de la vida, en el que la significatividad de los aprendizajes no puede resultar cuestionada. Y esto a pesar de que los riesgos de éste constituyen, en realidad, un entorno apropiado solamente para el ser humano maduro. (No obstante, hay que notar que el hecho de que la educación se prolongue durante toda la vida, sin alcanzar nunca un término o meta final, es decir, sin abandonar nunca una cierta situación transitoria, procesual, que nunca deja de ser precaria en cierta medida, hace no sólo difícil, sino imposible establecer distinciones de este tipo más que en un sentido metafórico). De aquí que las salidas del centro son recibidas de manera tan positiva por los estudiantes y les resultan sumamente alentadoras (Ferraz Lorenzo recoge los numerosos beneficios corporales, morales e intelectuales de las colonias, centrándose en el caso de Canarias) (Ferraz Lorenzo y Alonso Delgado, 2014). 
Por tanto, nos encontramos ante una especie de paradoja, según la que el joven más mediado tecnológicamente de la historia, el que consume gran porcentaje de su tiempo detrás de una pantalla, se manifiesta de la manera más patente (a través de un flagrante índice de fracaso escolar o del desprecio del trabajo académico) como necesitado de contacto real y directo con el mundo de la práctica; quiere vivirse, experimentarse en acción en el mundo real. Reconocer esta necesidad apremiante constituye el primer paso que el profesorado tiene que dar para dar respuesta a la situación que vive el estudiante y facilitarle su proceso educativo.

\subsection{La multiplicación y potenciación de resultados educativos a través del contexto informal}

Además, la transmisión educativa que tiene lugar en contextos informales presenta la virtualidad de que se autopotencia, es de alguna manera difusiva. Ante un sujeto abierto, receptivo, atento, despierto y reflexivo, tiene de alguna manera un poder generativo, multiplicador de los resultados. El espíritu de este tipo reconoce todo lo que se presenta como oportunidad de aprendizaje. Es selectivo, detecta lo que tiene valor, lo que le sirve y, en una transformación a través de la que lo hace suyo, lo incorpora y aprovecha, lo convierte en un resultado -a veces difuso, pero no por difuso o provisional menos presente- que se vuelve a poner en juego, vuelve a intervenir o se reinvierte en futuros contextos u ocasiones de aprendizaje. Esto puede representarse gráficamente en la imagen de una espiral continua, cuyo dinamismo, cuyo movimiento constante supone una superación de las anteriores perspectivas, una modificación continua que dura toda la vida, y en la que la vuelta a la consideración del punto de partida no supone nunca el regreso al mismo punto desde el que se partió.

Hablar de este fenómeno no es otra cosa que hablar de la experiencia y reparar en el enorme valor que tiene. Decía Inciarte que el modo de ser característico del ser humano es análogo al de un caracol que lleva su casa a cuestas, o al de un árbol que retiene toda su existencia anterior en los anillos grabados en su tronco, en el sentido de que retiene el pasado vivido y proyecta lo que está por venir: "La sustancia física es, en virtud de su temporalidad, análoga a un caracol que carga en sí todas sus pertenencias -omnia mea mecum porto-, o como un árbol que tiene sus anillos dentro de él» (Inciarte, 2005). Este comportamiento temporalizador (que asume y proyecta) es lo característico de la experiencia humana del mundo y de uno mismo.

A nadie se le escapa, y menos hoy en día, el importante activo que supone el hecho de ser experimentado. Pero ya Aristóteles había reparado, de hecho, en él:

La experiencia parece ser casi de la misma naturaleza que la ciencia y el arte; pero la ciencia y el arte llegan en los hombres a través de la experiencia, porque "la experiencia creó el arte, y la inexperiencia el azar» como dice Polo. El arte nace cuando de una multitud de nociones empíricas se separa un solo juicio universal aplicable a todos los casos semejantes. En efecto, formar el juicio de que tal 
medicina ha curado a Calias, enfermo de esta enfermedad, y después a Sócrates, y después a muchos otros tomados individualmente, es propio de la experiencia. Pero juzgar que tal medicina ha curado a todos los individuos afectos de tal enfermedad, determinados según una misma especie, como los flemáticos, los biliosos o los que tienen fiebre, corresponde al arte.

Ahora bien, en la vida práctica, la experiencia no parece diferenciarse en nada del arte. Incluso vemos que los hombres de experiencia superan a los que tienen el concepto (logos) sin la experiencia (Aristóteles, 1998, 71).

La experiencia se caracteriza por su carácter particular y contingente, es decir, está referida al aquí y ahora y a lo que podría ser de otra manera o incluso dejar de ser. Frente a ella, de la ciencia -y de la técnica derivada de ella- es propio el conocimiento del universal. Aristóteles afirma que, en consecuencia, los animales inferiores, y no sólo los humanos, poseen cierta experiencia ${ }^{7}$, pero lo que se escapa al alcance del animal irracional y es específico del ser humano es la ciencia y la técnica, que implican el conocimiento de lo general. Aunque la experiencia se confunde en ocasiones con éstas, pareciendo ambas indistinguibles por sus resultados, en realidad no son lo mismo ni pueden, por tanto, identificarse.

Pero lo que aquí nos interesa es la pregunta por aquello que caracteriza al sujeto experimentado, al que se toma la experiencia en serio y no simplemente la deja pasar. Es otra forma de preguntar: ¿qué le pasa a ese sujeto que sabe aprovechar el contexto informal, que incorpora verdaderamente la experiencia como ocasión para el aprendizaje? ¿Y qué hace falta, cuáles son las condiciones que permiten que tenga lugar tal aprovechamiento? En un segundo momento, ¿cómo se puede fomentar esto en la práctica educativa formal, para que a su vez los aprendizajes formales tengan repercusión, agiten al alumnado en la vida real? No hay que olvidar casos que se han dado de estudiantes de un curso de ética que por la mañana sacaban excelentes calificaciones, y eran arrestados por la tarde por cometer actos de vandalismo. Casos como éstos saltan la voz de alarma acerca del hecho de que algo falla en la escuela, que se ha alejado tanto de la vida real que es capaz de crear perfectos esquizofrénicos.

\section{LA REFLEXIVIDAD COMO CONDICIÓN DE POSIBILIDAD DEL APROVECHAMIENTO EDUCATIVO DEL CONTEXTO INFORMAL}

La condición de posibilidad o el requisito del aprovechamiento de las virtualidades educativas de los contextos informales radica en el desarrollo de estrategias metacognitivas, el impulso de la capacidad de aprender a aprender que consiste, en parte al menos, en reparar en el hecho de que todo -la realidad, los demás, las

7. "Los animales están dotados de sensación, y algunos de memoria e imaginación, y participan poco de la experiencia” (ARISTÓteles, 1998, 70). 
situaciones- se presenta como ocasión para $m i$ aprendizaje y en detectar cómo funcionan los mecanismos que me llevan a él. Esto es, se trata de reconocer mi propio proceso de afrontar el mundo, lo que me sale al encuentro -enfatizando el carácter singular y concreto, situado, contextualizado de cada individuo humano que recorre un camino de aprendizaje-. Si bien se puede alcanzar una cierta generalización en los procesos, se ha de partir del reconocimiento del hecho de que todos los caminos de aprendizaje son distintos (en velocidad, profundidad, amplitud), en la medida en que las personas lo son (Tourón, 2012), por lo que el desarrollo de los talentos también ha de serlo.

Es decir, hace falta reconocer no sólo de manera absoluta, simpliciter, el significado del fenómeno que se presenta (pregunta que, por cierto, no resulta tampoco nada fácil de responder), sino de detectar su significado en relación con la propia subjetividad. No sólo: ¿qué significa?, sino: ¿qué significa para mí, en mi vida particular y concreta, para el contexto en el que vivo?, ¿cómo me posiciono y hago frente a esto que me aborda?, ¿cómo lo trato, accedo a ello en cada uno de sus aspectos? En otras palabras, se trata de reconocer aquello no sólo en su dimensión objetivodescriptiva, sino en su relación con la propia conciencia, encuadrándolo, insertándolo en el curso de las propias experiencias vitales, y dotándolo de sentido en el conjunto de ellas. Dicen, por ejemplo, Gallego Ortega, García Guzmán y Rodríguez Fuentes que "para ser eficaz en la resolución de tareas, además de poseer un nivel suficiente de funcionamiento cognitivo, se requiere un adecuado control sobre el mismo» (Gallego Ortega, García Guzmán y Rodríguez Fuentes, 2014, 48).

Esta pregunta por la relación del fenómeno que se presenta con la propia trayectoria del sujeto es lo que debe fomentarse y lo que no puede faltar desde la enseñanza formal de ninguna disciplina. Hay que construir de manera explicita desde el aula esta conexión, para favorecer su construcción autónoma posterior, para salvar el salto entre el mundo académico y el vital del alumno concreto. Cada vez es más frecuente encontrar universitarios que no hilan o conectan lo aprendido en el mundo académico con las experiencias vividas en el ámbito de la práctica profesional. Éste es el punto central que ha de abordarse, el gran problema al que se enfrenta la enseñanza, en tanto que esa conexión es lo que dota de profundidad a la educación, lo que la aleja de una práctica memorística o histórico-doxográfica y la convierte en un ejercicio de pensamiento, reflexión y crítica. Sokolowski habla de cómo las palabras no sólo son signos de que el hablante está llevando a cabo la intensa actividad del lenguaje, de predicar algo de un fenómeno, sino que también son signos que invitan al oyente $a$ hacer lo mismo, a reconstruir, siguiendo la estructura de la frase, aquel pensamiento, a seguir ese curso de pensamiento, a llevar a cabo la actividad de predicar que, al principio, es necesariamente guiada, pero que después puede desembocar en distinciones nuevas y usos creativos del lenguaje (Sokolowski, 1978). Hasta se atreve a decir que «una persona no ha hablado hasta que no ha sido entendida» (Sokolowski, 1978, 116), apuntando con esto a la necesidad de la confluencia de las actividades de comunicador y receptor. La educación no puede limitarse a ser pura recepción, sino que se trata de que el estudiante cree e invente a partir de lo recibido o dado en la tradición transmitida 
por el docente. Hay verdadera comunicación educativa cuando el estudiante transforma, siquiera sea mínimamente, lo que recibe en una nueva comprensión (no necesariamente un producto). Esa comprensión es abierta: individual, distinta, novedosa en cada caso, en tanto que en ella entran en juego elementos de su propia experiencia.

\section{LA CAPACIDAD REFLEXIVA COMO EQUILIBRIO DE DISTANCIA Y CERCANÍA}

El desarrollo de la capacidad de aprender a aprender que puede llevar a explotar el potencial educativo de los contextos informales va emparejado, por tanto, con la reflexividad del sujeto. Ésta se traduce en lo siguiente: por una parte, cierta distancia que me permita enjuiciar la situación, que es necesaria para poder separarme de mí mismo (de mis intereses concretos, inclinaciones o apetencias particulares respecto de ella) y comprenderme como objeto, y hacer lo mismo respecto a la situación que se presenta en cuestión (el objeto es precisamente lo que está delante, lo que está 'ante' mí y no 'en' mí, y que es, en consecuencia, lo radicalmente otro al sujeto). En uno y otro caso, el del yo y la situación en que se encuentra, se trata, por una parte, de un curso concreto de pensamiento de la subjetividad y, por otra, de una situación particular, con características individuales que le son propias y que voy reconociendo progresivamente. Pero estos rasgos particulares de cada uno de ellos se abordan desde un comportamiento objetivador, siempre abierto a la posibilidad de extrapolar aquello en leyes universales.

Por otra parte, el comportamiento reflexivo requiere la cercanía suficiente para reconocerme (fáctica o hipotéticamente) en el actor o situación que vivo o tengo delante, para entenderme como afectado, implicado en ella (en caso de que fuera otro el implicado, lo reconozco a él y me reconozco a mí mismo como sujeto racional en general, comprendiéndolo como otro-yo).

Es decir, se trata de un juego, un equilibrio entre cercanía y distancia, identidad y diferencia: cercanía que, por una parte, no supone la inmediatez de la vida o de la acción cotidiana, o la plena implicación o identificación vivencial, la indistinción del yo respecto de aquello que se aparece que impida la labor de crítica o la comprensión de aquello en su aspecto de universalidad; y distancia que, por otra parte, no elimina la posibilidad de concebir aquel objeto en su relación conmigo.

\section{El SER humano, a la Altura DE LA NOVEDAD Y RAÍZ DE NOVEDAD}

La capacidad reflexiva es la que permite al ser humano redirigir constantemente su acción, tomando un curso posible de ella u otro. Es lo que le hace modificar constantemente el propio hacer conforme a lo experimentado, reformular sus conocimientos acerca de ello, atendiendo a las peculiaridades características de la situación que «salen al encuentro", de manera muchas veces inesperada, y que llevan al replanteamiento o reajuste de la manera de abordar aquello. Es la capacidad constante y continua de corrección -el conocimiento en la acción, no simplemente 
sobre ella, es decir, que se desarrolla y despliega mientras ésta dura y conforme a las características que va presentando, el aprender haciendo del que habla Schön inspirándose en Dewey y que hemos visto más arriba (Schön, 2010)-, aquella de la que no está dotada lo mecánico, pues, aunque esto es capaz de revisar sus procedimientos hasta cierto punto (hasta el punto en que el humano haya previsto), a veces la realidad supera las expectativas, y es el sujeto humano el único que puede estar a la altura de la novedad en la apertura de sus respuestas, creando interpretaciones antes no inventadas o previstas.

Esa capacidad abierta, ilimitada de reacción, raíz de la reflexividad humana, por la que la conciencia se vuelve afuera y adentro una y otra vez, y se hace cargo tanto de la circunstancia como de sí misma, combinando y ajustando ambas esferas fuera del esquema técnico naturalista de la acción-reacción, es lo que caracteriza al conocimiento específicamente humano frente a lo que hacen las máquinas. Las respuestas a estímulos, aunque puedan ser muchas, e incluso aleatorias, son siempre limitadas, determinadas, de modo que, en el caso de lo puramente técnico o mecánico, en la aplicación de reglas, queda excluida de raíz la novedad, la posibilidad del genio característica de la especie humana.

Por este carácter reflexivo típicamente característico del sujeto e implicado necesariamente en todo proceso de aprendizaje, y por la índole novedosa de las respuestas humanas, la asimilación que cada uno haga de la enseñanza recibida, es decir, el efecto que tenga la actividad del profesor en el alumno es único en cada caso. Aquí está la fuente y la condición de posibilidad de la creatividad, la imposibilidad de que el conocimiento sea explicado o «deducido» a partir de causas. Así se puede decir que, en último término, el comprender en sentido amplio -ese comprender apropiador (de un modo de vida, de esa dimensión vivencial de la enseñanza, no sólo de la puramente teórico-técnica) en que consiste el aprendizaje con la guía de un maestro- es algo que tiene que realizar cada uno. Si bien la metáfora del comer, de la ingestión, no es adecuada para explicar la actividad del conocimiento (en tanto que el objeto conocido no resulta alterado, modificado o destruido al conocerlo, frente a lo que ocurre al ingerir un alimento), sí se puede decir que, al igual que uno no se puede comer una cosa por otro, tampoco puede conocerla por él, sino que esto es una actividad de la autonomía humana.

Por suerte, el saber (al igual, por cierto, que el bien o el mal) no es algo que se pueda dar, que se pueda traspasar de uno a otro de manera puramente mecánica. Es decir, no es ninguna 'cosa', sino que es lo que queda tras recorrer un camino, tras haber adoptado unos métodos y, en último término, hábitos o modos de vida. Y lo que se genera a partir de éstos resulta ser siempre abierto, imposible de prever o novedoso. Por eso toda concepción puramente técnica del conocimiento, que encarna de manera exclusiva a éste, por ejemplo, en la adquisición de unas competencias, no es suficiente, pues, por un lado, no concibe o no permite explicar la posibilidad de la creatividad y, por otro, no pone el énfasis en el punto correcto y pierde o confunde parte de su esencia, en tanto que no tiene en cuenta esa dimensión vivencial que está implicada en la actividad educativa y que se cifra en la educación moral. 
El feedback se advierte aquí como fundamental para el desarrollo de esta capacidad reflexiva y autorreguladora del estudiante, que va emparejada con la potencia creativa. Éste ha de ser, además, comprendido de manera no simplista y unidireccional (de profesor a alumno), sino como co-construido, de modo que en esta tarea participan también otros estudiantes. Cano García analiza la evolución que ha experimentado este término (Cano García, 2014). Se habla incluso de feedforward para enfatizar el sentido proyectivo que ha de tener para ser efectivo, como guía, retroalimentación que el estudiante ha de incorporar en las tareas subsiguientes. Pero esta transformación requiere de un cambio en la actitud de los alumnos y alumnas (y, en muchos casos, de los propios docentes): un educando no centrado en los resultados, habitualmente expresados en la forma de calificaciones numéricas, sino en su propio proceso de aprendizaje: en su situación dentro de éste. Es decir, un estudiante enfocado en progresar en su propio camino individual de desarrollo de su propio talento. El criterio de medida de este progreso individual no es la nota obtenida en general o en relación con el resto de compañeros y compañeras, sino el avance logrado desde su particular punto de partida en cada una de las materias.

\section{REFLEXIVIDAD E IDENTIDAD HUMANA}

No podemos dejar de notar que el desarrollo de la competencia de aprender a aprender, la mejora de la capacidad reflexiva es, en último término, un avance en la formación de la propia identidad, una evolución en la conciencia y comprensión de uno mismo y de su posición respecto al conjunto de lo real. No olvidemos que la educación debe, si no dar respuesta, sí plantear, al menos en la medida en que le es posible, las preguntas acuciantes que afectan a todo ser humano y que constituyen la raíz que está a la base de muchas expresiones u objetivaciones artísticas y científicas. El hombre es un ser necesitado de preguntas. Se podría decir que la crisis intelectual más grave se vive cuando el hombre deja de planteárselas, y para ello es esencial dar buenos ejemplos de ellas y enseñar a formularlas (Heidegger, 2008). Toda investigación, todo trabajo humano, todo esfuerzo empieza por una pregunta y, mientras el hombre se las plantee, de alguna manera estará construyendo respuestas.

Cuando expresamente se oculta, o cuando, más habitualmente, por descuido, no se aborda explícitamente esta base, se corre el riesgo de no mostrar bien al estudiante la fuente última, el origen de los problemas o asuntos que han ocupado al hombre y que constituyen diversos rasgos o aspectos de su cultura. Hay que construir explícitamente ante el estudiante el nexo, la conexión entre los productos culturales o científicos y su porqué para ayudarle a dar sentido, a encajar significativamente las diversas piezas del engranaje que constituye el conocimiento y ayudarle a formar una visión del mundo coherente, sin lagunas; es decir, no formada por ámbitos inconexos.

Esas preguntas básicas, preguntas-raíz, se plantean no sólo en el ámbito de la educación formal, sino que la vida es una gran maestra en este sentido. 
Precisamente el riesgo que se vive hoy en día es el dejar de abordarlas de manera explícita en el contexto formal. En ese momento se agranda más la distancia entre el mundo de la escuela, el aula de la institución educativa, y el mundo de la vida.

\section{LA INMEDIACIÓN COMO OBJETIVO O FIN ÚLTIMO DE TODA MEDIACIÓN EDUCATIVA}

Así pues, reparamos en el hecho de que la educación formal es necesariamente signo, comunicación (Altarejos y Naval, 2011), lenguaje, mediación, pero el fin de la acción educativa es que el estudiante alcance la inmediación: la captación, la comprensión o el hacerse cargo de los diversos procesos y estructuras de lo real. Esta inmediación se consigue en el concepto. En él se da la máxima cercanía con lo real, más que en la tenencia meramente física del objeto. Ése es el reto del educador: a través de una mediación (el lenguaje, la imagen y todo tipo de signos materiales encarnados en los medios pedagógicos que tiene a su disposición) lograr la presencia más completa de la realidad que se da en el concepto. Es decir, ayudar al alumno o alumna a formar conceptos; a través del lenguaje, de recursos educativos de variado tipo, suscitar pensamiento.

El contexto informal tiene gran potencial educativo, como hemos visto más arriba, por su inmediatez, aunque hay que observar que la inmediatez que éste ofrece no es la del concepto, sino la de la implicación vivencial total en él del sujeto. Lo que hace falta para convertir la experiencia del contexto informal en concepto (elevarla a concepto, podríamos decir -y, por tanto, dotarla de un carácter de universalidad-), para aprovecharla como ocasión para el aprendizaje, es una cierta distancia: la que resulta de aplicar a ella la capacidad reflexiva del sujeto, de poner en juego su reflexividad. Ésta es la que hay que potenciar en el contexto formal para sacar partido del contexto informal y multiplicar la acción educativa que el estudiante recibe: practicar en el aula los mecanismos para desarrollar sujetos reflexivos, tratar explícitamente la exploración guiada de la propia subjetividad al enfrentarse a contenidos, situaciones u otros sujetos, pues esto es lo que permite directamente el desarrollo y la regulación del autoconocimiento, la adaptación del sujeto a contextos imprevistos y, en definitiva, la adquisición y potenciación de la competencia de aprender a aprender.

\section{EL PROCESO DE LA EDUCACIÓN MORAL EN CONTEXTOS INFORMALES}

Podemos retomar la cuestión que nos planteábamos al principio, en el primer apartado, cuando nos preguntábamos cómo acontece la transmisión, en este caso, educativa y, en concreto, referida a cuestiones morales. Es decir, cómo tiene lugar la incorporación de lo moral al propio ser del que comprende, ciñéndonos, además, a los contextos de aprendizaje informal. En otras palabras, y para dar respuesta a la cuestión central que plantea el título del presente artículo, ¿cómo se lleva a cabo el aprendizaje moral en la cotidianeidad de la vida? ¿Cuál es el mecanismo de la 
educación moral en este tipo de contextos? ¿Por qué resultan éstos educativos en este sentido? Pues bien, podemos afirmar que, en último término, la educación moral está operativa en ellos a través del planteamiento (de la transmisión -que se da, como vimos, de manera necesaria, incluso de forma involuntaria o contra la voluntad del sujeto-) de un modo de vida por parte de los implicados en la situación, y que, si bien no resulta nunca perfectamente coherente, en vistas del carácter complejo de la naturaleza humana, no puede decirse tampoco que carezca completamente de coherencia, es decir, resulta coherente de manera limitada o en cierto grado. Esto es, el aprendizaje moral está aquí presente por la propuesta de un modo de vida que se da esos contextos, que es efectivamente transmitida, ya sea de manera explícita o expresa (es habitual encontrar en la cotidianeidad, en los contextos de la vida, quien expone o justifica su modo de vivir, o anota sus preferencias en el sentido de sus opciones de vida) o de forma implícita u operativa, a través de los actos de los agentes implicados ${ }^{8}$.

Ese modo de vida está configurado y es manifestativo o denotativo de una jerarquía de bienes (fines), de manera que está dotado de una cierta unidad: la unidad del fin último que se persigue, que es reconocido por el que presenta tal proyecto de vida como valioso en sí mismo, y no meramente como un medio o instrumento para otra cosa (Aristóteles, 1998) y, por tanto, como deseable en sí mismo y configurador de un deber para el sujeto. Sirve, así, de fundamento o base para justificar un precepto, ley o mandato que obliga a buscarlo y preservarlo de manera incondicionada, es decir, sin relación a ninguna otra cosa. Como afirma Aristóteles, el fin es lo que da sentido a la acción, por lo que, para comprender el sentido de algo, he de acudir a aquello que lo motiva, y esto se encuentra ya en el principio de la acción (entendiendo "principio" en sus dos sentidos de inicio y fundamento). Esto es, hay que entender la acción como un proyecto regido desde el principio por un fin y que tiene a éste como principio: "Todo arte y toda investigación e, igualmente, toda acción y libre elección parecen tender a algún bien; por esto se ha manifestado, con razón, que el bien es aquello hacia lo que todas las cosas tienden" (Aristóteles, 1998, 131). De este modo, no entiendo una acción si no entiendo su porqué, su sentido, y esto puede aplicarse a la vida humana en su conjunto.

Decíamos arriba que cualquier modo de vida mantenido no llega a ser plenamente coherente en el caso humano, y esto resulta claro por la propia experiencia: reorientamos nuestras acciones, no sólo a nivel particular o individual, sino también en sentido global o total: cambiamos nuestras creencias más permanentes o

8. A veces la transmisión efectiva u operativa de esos modos de vida, imbricados en la forma de ver la realidad y afrontar la vida que se trasluce en cada uno de los actos, es más efectiva, pedagógicamente hablando, que su predicación o exposición explícita. Con un ejemplo, esto se aprecia bien en el caso del párroco don Manuel, en San Manuel Bueno, mártir, que es más elocuente en lo que muestra (su agonía al afrontar los momentos de soledad, sus silencios en medio de las oraciones) que en lo que dice: la fe que constantemente profesa. 
de fondo a medida que nos vamos configurando con el tiempo y, en consecuencia, se producen con cierta frecuencia choques en nuestro propio pensamiento y sentir. No cabe duda de que no es humanamente posible una perfecta coherencia en el propio proyecto vital, al igual que al hombre le resulta imposible lograr una total autotransparencia, en el sentido de una completa apropiación del yo individual y sus motivos. Nada hay más característicamente humano que el conflicto interno.

Ya Platón, intentando retratar el alma humana u ofrecer las pinceladas esenciales para la comprensión de quién es el hombre, dijo que no se podía responder a esta pregunta de manera directa, por considerar esto tarea más bien divina, pero sí podía arrojar luz sobre el asunto a través de un mito, el del carro alado. En éste se pone de manifiesto la tensión específicamente humana que afecta al hombre de manera permanente: su deseo y tendencia irreprimible a lo divino, la aspiración a lo absoluto e infinito, a perdurar y no dejarse morir (de hecho, Aristóteles considera que no aspirar a aquello de lo que se es capaz resulta indigno) (Aristóteles, 1994) y, a la vez, su realidad radicalmente limitada, su carácter finito y mortal, que va acompañado de una cierta tendencia a la tierra, de la seducción por el atractivo o la ilusión de los bienes aparentes pasajeros.

Cierto grado de tensión es connatural al alma humana. No obstante, el grado de incoherencia en el estilo de vida efectivamente defendido por algunos ha llegado a ser tan extremo que podría afirmarse que la incoherencia misma ha llegado a constituirse en modo de vida. En este sentido, no resulta extraño escuchar por ahí que uno es, de lunes a viernes, un cierto tipo de persona, y durante el fin de semana se transforma en otra. Hoy soy una persona, mañana otra; por la mañana soy de un modo y por la noche me convierto en un tipo distinto. El cambio de identidad, incluso convertido en práctica cotidiana, queda incluido dentro de las posibilidades humanas. Sin embargo, las metamorfosis temporales o pasajeras, las actitudes esquizofrénicas o los cambios radicales instaurados en habitualidad no suelen serlo tanto en la práctica, pues en ésta no cabe eludir, al fin y al cabo, la presencia de un fin o bien último, que cabe ciertamente modificar o comprender de otro modo, pero no sustituir de manera drástica a cada instante. No es viable el permanente tránsito entre modos de vida contradictorios, en la medida en que están regidos por lo que en cada uno de ellos se considera como el bien máximo de la vida, el contenido concreto de la felicidad (respecto a la cual Aristóteles decía que todos coinciden en el nombre, pero no en aquello en que consista, puesto que cada uno la comprende de distinta manera) (Aristóteles, 1998). En tanto que éste, el fin último, tiende a presentar una cierta permanencia o invariabilidad, el proyecto vital ha de ser hasta cierto punto (dentro de la natural variabilidad humana -recuérdese el aforismo clásico: la ratio humana es, en realidad, correcta ratio-) también unitario.

Estas condiciones o modos de vida que se ofrecen a la percepción y al análisis informal en la cotidianeidad de la vida constituyen la fuente del aprendizaje moral en tanto que expresivas de la tendencia a un fin último concreto, de la consideración de algo como bien supremo, consideración con la que uno puede sentirse 
identificado y llegar a asumir como propia, o de la que uno puede disentir de modo no planificado ni académico.

Son muchas las operaciones intelectuales y valorativas que se ponen en juego en este tipo de contextos, los informales. A saber, la captación y comprensión global de un proyecto de vida ajeno; el examen de la jerarquía no explícita de bienes en él; el análisis espontáneo, no planificado o no formal de los actos expresivos de aquél; la detección del grado de coherencia o armonía y de la racionalidad implícita en ellos percibida desde fuera, y la comparación valorativa de todo esto con el propio proyecto vital. Todas estas acciones constituyen en gran medida los mecanismos de operatividad o la lógica que se pone en juego en los contextos informales, por la que se hace efectivo el aprendizaje moral en ellos. Y llama la atención lo semejantes, e incluso idénticas en algunos casos, que son estas operaciones mentales respecto a las que tienen lugar en el estudio formal de las diversas materias en contextos institucionales. Así pues, se ofrece aquí otro motivo incuestionable de la necesidad de hacer un esfuerzo mayor para lograr un acercamiento mayor entre ambos tipos de contextos que multiplique sus resultados.

Por tanto, hemos visto que la transmisión de modos de vida en todo tipo de contextos se da de manera necesaria, y ésta cristaliza en operaciones encaminadas a la comprensión y, en consecuencia, en aprendizaje moral cuando tiene lugar, por parte del aprendiz, una transformación apropiadora de ese modo de vida. De esta manera, se abre la posibilidad de que este modo de vida pase a convertirse, en último término, en norma, al descubrir las razones de él en relación con la propia subjetividad.

En definitiva, la educación moral significa en su sentido más amplio que, ya sea desde un contexto formal o desde otro informal, se produce efectivamente una transmisión que se comprende como propuesta por parte de un sujeto (no sólo por parte del docente) hacia otro, en el sentido etimológico de pro-posición ('poner adelante, para que otras personas tomen conocimiento de una intención'). Pues habitualmente, dejando de lado algunas excepciones, la adopción o incorporación de algo -de un estilo de vida, por ejemplo- o su formulación explícita son signos de que aquello se secunda o se acepta (no hay demostración más clara de que soy partidario de algo o de que tengo una intención que cuando lo hago o lo digo, en la mayoría de los casos). Esa transmisión o propuesta puede conducir a la adquisición por parte de otro sujeto -en la medida en que éste haya desarrollado su capacidad reflexiva- de ese modo de vida configurado por unos fines, lo cual lleva en último término a la universalización de la categoría de maestro y alumno.

\section{Conclusiones}

Se han planteado algunos recursos y competencias consideradas vitales o críticas para la formación humana, que se requieren para estar a la altura de los tiempos y que se ponen en juego no sólo en el aprendizaje en contextos formales, sino constantemente a lo largo de toda la vida, por ser el hombre no simplemente 
un ser de experiencias (pues hemos visto que se puede decir también que los animales las tienen), sino capaz de aprovechar o desaprovechar, aprender o no hacerlo de la experiencia, sacar el máximo partido o no de ella. Aquí se encuentra implicado, por tanto, el aprendizaje a partir de ellas en los contextos informales.

Hemos visto que Inciarte (Inciarte, 2005) decía, empleando una metáfora, que el ser humano puede asimilarse a un caracol o a un árbol en tanto que lleva consigo el pasado, lo incorpora y es, además, el único que puede proyectar el futuro (Millán-Puelles sostiene también que la vida humana sería inconcebible sin toda la dosis de irrealidad que está implicada en la representación humana del pasado y el futuro). Pero, pedagógicamente hablando, hay que decir que esos anillos del árbol o esa carga que el caracol lleva a cuestas no sirven igual para todos: hay que saber aprovecharla, aprender de esa experiencia. Hay que notar que la pura acumulación de experiencias, el paso del tiempo, tiene un potencial educativo limitado. Es cuando el sujeto sabe encarar aquello que vive o a lo que se enfrenta (es crítico, lo selecciona, lo compara respecto de sí mismo, sabe escoger lo que quiere como modelo, no todo lo da por bueno) cuando esto se convierte en un instrumento potente fuente de numerosos aprendizajes. Es decir, se trata de saber cómo afrontar la experiencia, cómo convertir las experiencias en recursos valiosos de aprendizaje.

Para ello hemos determinado la reflexividad (que viene marcada por esa ambivalencia, ese equilibrio entre identidad y diferencia, cercanía y distancia) como la capacidad básica o fundamental que el educando necesita, cuyo cultivo lleva al desarrollo de la competencia de aprender a aprender: el reconocimiento y la selección de aquello que me interesa para incorporarlo de alguna manera a la propia vida, basado en la comprensión de que todo se presenta como ocasión posible de aprendizaje. El desarrollo en el aula de estas destrezas capacita al alumno o alumna para aprovechar el enorme potencial educativo de los contextos informales, cuya importancia no puede pasar desapercibida al docente, en tanto que éstos son constantemente operativos.

En definitiva, desde el ámbito formal hay que enseñar a cultivar las capacidades que nos permiten sacar partido de las ocasiones de aprendizaje informal, que desbancan en número y frecuencia a las de aprendizaje en contextos formales. Ésta es la integración o convergencia que autopotencia el aprendizaje. Y la base que constituye la condición de posibilidad de esta traducibilidad, de esta convergencia o afinidad entre ambos tipos de contextos es el hecho de que ambos comparten un mismo tejido moral. En los dos está operativo el carácter moral, susceptible de ser aprendido, que es intrínseco a las acciones humanas.

\section{REFERENCIAS BIBLIOGRÁFICAS}

Altarejos, F. y Naval, C. (2011) Filosofía de la educación. Pamplona, Eunsa. ARISTóteles (1974) Poética. Madrid, Gredos. ARISTÓTELEs (1994) Metafísica. Madrid, Gredos. ARIstóteles (1998) Ética a Nicómaco. Madrid, Gredos. 
Cano García, E. (2014) Análisis de las investigaciones sobre feedback: aportes para su mejora en el marco del EeEs. Bordón. Revista de Pedagogía, 66 (4), 9-24.

Ethel, R. y McMeniman, M. (2000) Unlocking the Knowledge in Action of an Expert Practitioner. Journal of Teacher Education, 51 (2), 87-101.

Ferraz Lorenzo, M. y Alonso Delgado, V. (2014) Antecedentes, surgimiento y desarrollo de las colonias escolares de vacaciones en Canarias. Bordón. Revista de Pedagogía, 66 (4), 25-37.

Gadamer, H. (1988) Verdad y Método. Fundamentos de una hermenéutica filosófica. Salamanca, Sígueme.

Gallego Ortega, J. L.; García Guzmán, A. y Rodríguez Fuentes, A. (2014) Estrategias metacognitivas en la expresión escrita. Bordón. Revista de Pedagogía, 66 (4), 39-53.

GeHLEn, A. (1980) El hombre. Salamanca, Sígueme.

GreEn, T. F. (1985) The Formation of Conscience in an Age of Technology. American Journal of Education, 94 (1), 1-32.

HeIdEgGer, M. (2008) Introducción a la investigación fenomenológica. Madrid, Editorial Síntesis.

HUSSERL, E. (1962) Ideas relativas a una fenomenología pura y una filosofía fenomenológica. México, Fondo de Cultura Económica.

IbáÑ̃zZ-MarTín, J. A. (2013) Ética docente del siglo XXI: Nuevos desafíos. Edetania, 43, 17-31.

InCiarte, F. (2005) First Principles, Substance and Action. Studies in Aristotle and Aristotelianism. Hildesheim/Zürich/New York, Olms Verlag.

Knight, S. L.; Lloyd, G. M.; Arbaugh, F.; Gamson, D.; McDonald, S. P.; Nolan, J. y WhitNEY, A. E. (2015) Reconceptualizing Teacher Quality to Inform Preservice and Inservice Professional Development. Journal of Teacher Education, 66 (2), 105-108.

Maxwell, B.; Tremblay-laprise, A.; Filion, M.; Boon, H.; Daly, C.; Van Den Hoven, M.; Heilbronn, R.; Lenselink, M. y Walters, S. (2016) A Five-Country Survey on Ethics Education in Preservice Teaching Programs. Journal of Teacher Education, 67 (2), 135-151.

Millán-Puelles, A. (1967) La estructura de la subjetividad. Madrid, Rialp.

SCHÖN, D. A. (2010) La formación de profesionales reflexivos. Hacia un nuevo diseño de la enseñanza y el aprendizaje en las profesiones. Barcelona, Paidós.

Sokolowski, R. (1978) Presence and Absence. A Philosophical Investigation of Language and Being. Bloomington, Indiana University Press.

Steiner, G. (2011) Lecciones de los maestros. Madrid, Siruela.

TOuRÓN, J. (2012) 5 razones por las que la igualdad produce injusticia. Javier Tourón: talento, educación, tecnología. Consultado el 25 de mayo de 2016.

http://www.javiertouron.es/2012/04/5-razones-por-las-que-la-igualdad.html.

TOuRón, J. (2012) El bachillerato de excelencia: iigualdad o equidad? Javier Tourón: talento, educación, tecnología. Consultado el 25 de mayo de 2016.

http://www.javiertouron.es/2012/04/el-bachillerato-de-excelencia-igualdad.html.

Unamuno, M. (2005) San Manuel bueno, mártir. Madrid, Cátedra. 